\title{
New magnetic phase in the nickelate perovskite $\mathrm{TINiO}_{3}$
}

\author{
L. Korosec, ${ }^{1, *}$ M. Pikulski, ${ }^{1}$ T. Shiroka, ${ }^{1,2}$ M. Medarde,${ }^{3}$ H. Luetkens, ${ }^{4}$ J. A. Alonso, ${ }^{5}$ H. R. Ott, ${ }^{1,2}$ and J. Mesot ${ }^{1,2}$ \\ ${ }^{1}$ Laboratory for Solid State Physics, ETH Zürich, CH-8093 Zürich, Switzerland \\ ${ }^{2}$ Paul Scherrer Institut, $\mathrm{CH}-5232$ Villigen PSI, Switzerland \\ ${ }^{3}$ Laboratory for Scientific Developments and Novel Materials, Paul Scherrer Institut, CH-5232 Villigen, PSI Switzerland \\ ${ }^{4}$ Laboratory for Muon Spin Spectroscopy, Paul Scherrer Institut, CH-5232 Villigen PSI, Switzerland \\ ${ }^{5}$ Instituto de Ciencia de Materiales de Madrid, CSIC, Cantoblanco, E-28094 Madrid, Spain
}

(Received 16 January 2017; published 16 February 2017)

\begin{abstract}
The $\mathrm{RNiO}_{3}$ perovskites are known to order antiferromagnetically below a material-dependent Néel temperature $T_{\mathrm{N}}$. We report experimental evidence indicating the existence of a second magnetically ordered phase in $\mathrm{TINiO}_{3}$ above $T_{\mathrm{N}}=104 \mathrm{~K}$, obtained using nuclear magnetic resonance and muon spin rotation spectroscopy. The new phase, which persists up to a temperature $T_{\mathrm{N}}^{*}=202 \mathrm{~K}$, is suppressed by the application of an external magnetic field of approximately $1 \mathrm{~T}$. It is not yet known if such a phase also exists in other perovskite nickelates.
\end{abstract}

DOI: 10.1103/PhysRevB.95.060411

Although first synthesized already in 1970 [1], the rareearth nickelates $\mathrm{RNiO}_{3}$ have been the subject of intense research efforts during the past decade due to their peculiar metal-insulator transition at $T_{\mathrm{MI}}$ and subsequent antiferromagnetic (AFM) order between Ni spins below a Néel temperature $T_{\mathrm{N}}[2,3]$. By substituting rare-earth ions $\mathrm{R}^{3+}$ with different radii, the perovskite lattice can be distorted continuously. This distortion affects the magnetic couplings of $\mathrm{Ni}$ spins by modifying the $\mathrm{Ni}-\mathrm{O}-\mathrm{Ni}$ superexchange angle. A phase diagram of $\mathrm{RNiO}_{3}$, mostly based on previously published results, is shown in Fig. 1. Despite recent theoretical [4-6] and experimental [7] progress, the nature of the paramagnetic (PM) insulating phase is still unclear. The AFM order below $T_{\mathrm{N}}$ is characterized by a propagation vector $k_{\mathrm{pc}}=(1 / 4,1 / 4,1 / 4)$ with respect to the (pseudo)cubic unit cell of the ideal perovskite structure. Thus, a period of the magnetic structure comprises four $\mathrm{Ni}$ sites along each pseudocubic crystal axis. Previous experimental reports suggested a collinear up-up-down-down structure [8-10], while others argued for a noncollinear spiral spin configuration [11-14]. The AFM phase is predicted to be ferroelectric $[15,16]$, but this has not yet been confirmed experimentally. Since the magnitude of the ferroelectric polarization is very different for the two candidate spin arrangements [16], investigations of the magnetism of $\mathrm{RNiO}_{3}$ are crucial for the understanding of their possible multiferroicity. Nuclear magnetic resonance (NMR) is a powerful technique to locally probe magnetic behavior. However, to our knowledge, the only previous NMR work involving a nickelate perovskite was a ${ }^{139}$ La NMR study of $\mathrm{LaNiO}_{3}[17]$, which is a PM metal at all temperatures.

In the present work, we present the first NMR investigation of an insulating member of the $\mathrm{RNiO}_{3}$ family, in the form of a combined NMR and muon-spin rotation ( $\mu \mathrm{SR})$ study of the magnetic properties of $\mathrm{TINiO}_{3}$. This material is known to have the same qualitative behavior as the analogous rare-earth nickelates [18]. From an $\mathrm{NMR}$ perspective, $\mathrm{TlNiO}_{3}$ is unique among the $\mathrm{RNiO}_{3}$ compounds because both ${ }^{203} \mathrm{Tl}$ and ${ }^{205} \mathrm{Tl}$ nuclei are excellent for NMR. In addition to the previously known AFM phase below $T_{\mathrm{N}}=104 \mathrm{~K}$, our measurements

*1korosec@phys.ethz.ch reveal a previously unknown magnetically ordered phase between $T_{\mathrm{N}}$ and $T_{\mathrm{N}}^{*}=202 \mathrm{~K}$. An applied magnetic field of $1 \mathrm{~T}$ is sufficient to suppress the static magnetic order between $T_{\mathrm{N}}$ and $T_{\mathrm{N}}^{*}$. Nevertheless, hysteretic dynamics in this temperature range are still detected by NMR, even in applied fields of several teslas (see below).

Experimental details. The synthesis of polycrystalline $\mathrm{TINiO}_{3}$ is described in Ref. [25]. We confirmed the $99 \%$ purity of our powder sample by x-ray diffraction. DC magnetometry data taken at various applied fields display a kink at $T_{\mathrm{N}}=$ $104 \mathrm{~K}$, consistent with the previously known AFM transition (see Fig. 2).

At low fields, a small ferromagnetic contribution with a Curie temperature $T_{\mathrm{C}}=25 \mathrm{~K}$ is observed, most likely due to the presence of residual $\mathrm{Ni}(\mathrm{OH})_{2}$ from the synthesis [25]. (Note that a similar feature can be seen in the susceptibility data shown in Refs. [18,25] as well.) Because of the large magnetic moment of this ferromagnet, an impurity concentration of $1 \%$ by mass is sufficient to explain the substantial magnetic response in our data. Due to the small mass fraction, this impurity is irrelevant for $\mu \mathrm{SR}$. Moreover, since this impurity does not contain thallium, it does not affect our ${ }^{203} \mathrm{Tl}$ and ${ }^{205} \mathrm{Tl}$ NMR measurements, either.

Nuclear magnetic resonance. ${ }^{203} \mathrm{Tl}$ and ${ }^{205} \mathrm{Tl}$ NMR spectra were acquired using a standard spin-echo pulse sequence. Because of the very large linewidth, the NMR signal in the AFM phase was acquired by sweeping the frequency and integrating the Fourier-transformed spin echo. The transverse relaxation time $T_{2}$ was measured by varying the delay $\tau$ between radio-frequency pulses in the spin-echo sequence and fitting the resulting NMR amplitudes to an exponential decay $M(\tau)=M_{0} \exp \left(-2 \tau / T_{2}\right)$. Due to the rapid relaxation and decoherence rates in the PM phase $\left(T_{1} \approx 20 \mu \mathrm{s}, T_{2} \approx 16 \mu \mathrm{s}\right)$, the spin-echo intensities are low and substantial signal averaging is required to improve the signal-to-noise ratio of the NMR measurements. In addition to standard NMR experiments in an applied field, we performed complementary zero-field NMR investigations in the magnetically ordered phase.

Two stable thallium isotopes, ${ }^{203} \mathrm{Tl}$ and ${ }^{205} \mathrm{Tl}$, occur naturally with abundances of $29.5 \%$ and $70.5 \%$. Both nuclei have spin $1 / 2$ and gyromagnetic ratios of $\gamma_{203} / 2 \pi=24.3216 \mathrm{MHz} \mathrm{T}^{-1}$ and $\gamma_{205} / 2 \pi=24.5603 \mathrm{MHz} \mathrm{T}^{-1}$, 


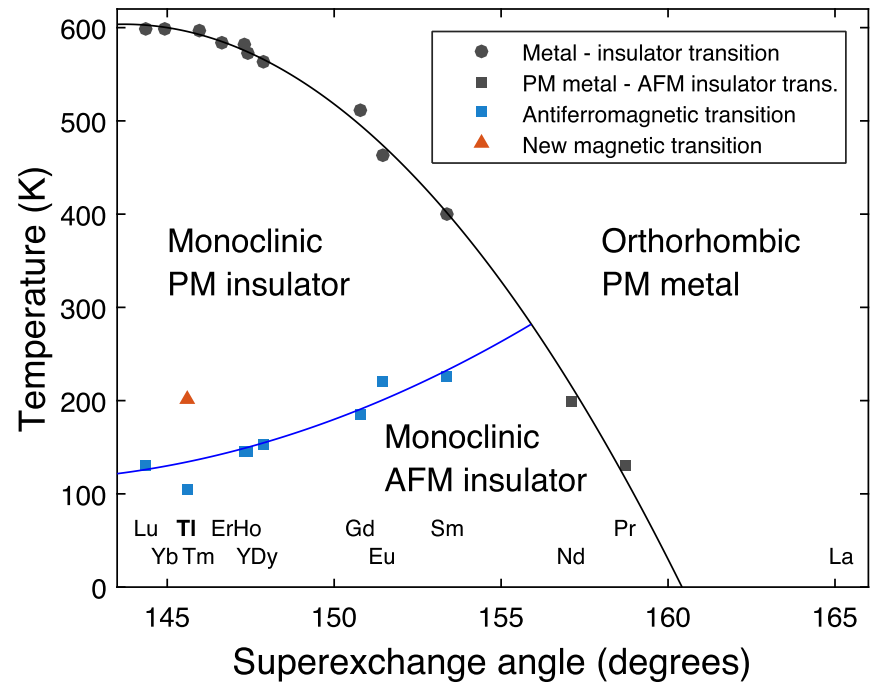

FIG. 1. Phase diagram of the $\mathrm{RNiO}_{3}$ perovskites with respect to temperature and Ni-O-Ni superexchange angle. Data for compounds other than $\mathrm{TlNiO}_{3}$ shown in this plot are taken from Refs. [2,19-24]. The red triangle indicates the new magnetic phase transition described in this work.

respectively. Due to the high abundances and gyromagnetic ratios, both nuclei are well suited for magnetic resonance measurements. In $\mathrm{TlNiO}_{3}$, the resonance signals of both ${ }^{203} \mathrm{Tl}$ and ${ }^{205} \mathrm{Tl}$ were detected at a frequency shift of $K=1.03 \%$ at room temperature (see Fig. 3).

Due to the distribution of orientations of the intrinsic magnetic field, the NMR powder pattern broadens by two orders of magnitude in the AFM phase. Two cusps, marked by vertical dashed lines in Fig. 3, are seen in the NMR spectrum, which

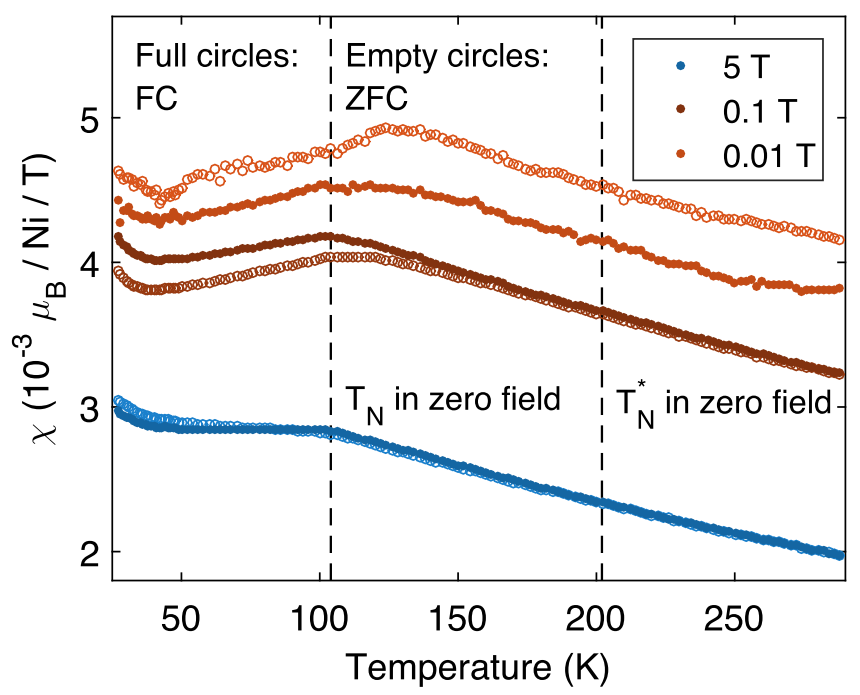

FIG. 2. Magnetic susceptibility of $\mathrm{TINiO}_{3}$ powder, measured using a commercial DC magnetometer at different magnetic fields. Vertical dashed lines at $T_{\mathrm{N}}=104 \mathrm{~K}$ and $T_{\mathrm{N}}^{*}=202 \mathrm{~K}$ indicate the magnetic transition temperatures in zero field, as determined by $\mu \mathrm{SR}$. The magnetic response below $25 \mathrm{~K}$ (not shown) is dominated by a ferromagnetic impurity contribution from unreacted $\mathrm{Ni}(\mathrm{OH})_{2}$ (see text for details).

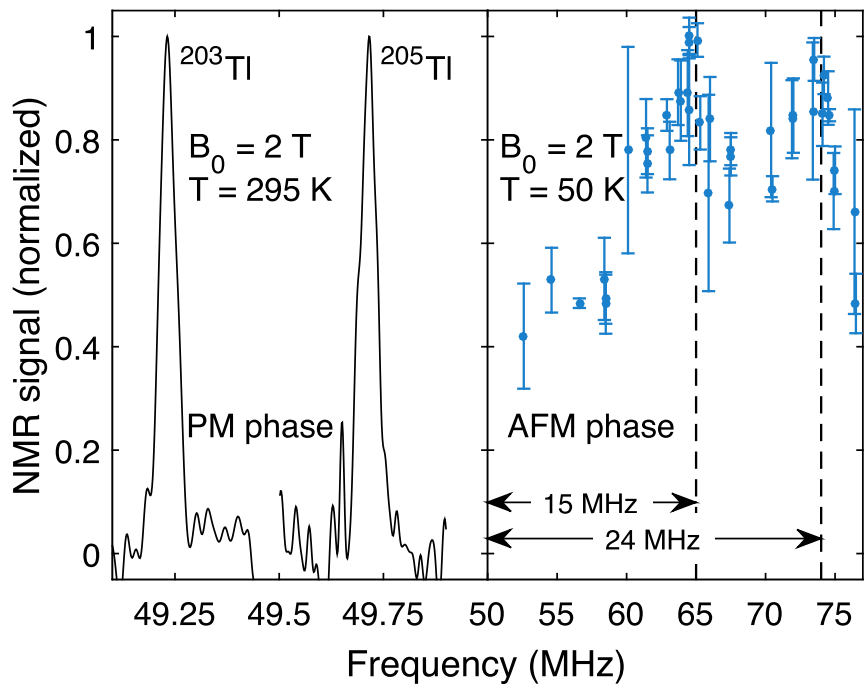

FIG. 3. ${ }^{203} \mathrm{Tl}$ and ${ }^{205} \mathrm{Tl} \mathrm{NMR}$ spectra at $295 \mathrm{~K}$ in the PM phase (left) and at $50 \mathrm{~K}$ in the AFM phase (right). Note the different frequency scales in the two panels. The broadened upper edges of two overlapping AFM powder patterns are emphasized by vertical dashed lines.

correspond to the broadened upper edges of antiferromagnetic NMR powder patterns from two magnetically inequivalent $\mathrm{Tl}$ sites. The edges originate from crystallites whose internal magnetic field is aligned parallel to the applied field [26]. They occur at frequencies

$$
v_{\text {edge }}=\frac{\gamma}{2 \pi}\left(B_{0}+B_{\text {int }}\right),
$$

where $B_{0}$ is the applied magnetic field and $B_{\text {int }}$ is the local internal field. This internal field is static on the typical time scale of an NMR experiment, which is at least $10^{-4} \mathrm{~s}$. Since the relative difference of the gyromagnetic ratios $\gamma_{205}-\gamma_{203} / \gamma_{205} \approx$ $1 \%$ is much smaller than the relative width of the powder pattern, the contributions from ${ }^{203} \mathrm{Tl}$ and ${ }^{205} \mathrm{Tl}$ cannot be distinguished in the magnetically ordered phase. Hence, when mentioning Tl NMR spectra in the AFM phase, we refer to the superposition of ${ }^{203} \mathrm{Tl}$ and ${ }^{205} \mathrm{Tl}$ NMR spectra.

By applying Eq. (1) to the NMR spectrum acquired at $50 \mathrm{~K}$ and $B_{0}=2 \mathrm{~T}$, shown in Fig. 3, we extract the two internal magnetic fields $B_{\text {int }} \approx 0.6 \mathrm{~T}$ and $1.0 \mathrm{~T}$. The zero-field (ZF) NMR frequency $v_{\mathrm{ZF}}=\gamma / 2 \pi B_{\text {int }}$ corresponds to $B_{0}=0$ in Eq. (1). Since $v_{\mathrm{ZF}}$ is proportional to the local magnetic field, its temperature dependence reflects the order parameter of a magnetically ordered phase.

By following the temperature dependence of the two ZF-NMR frequencies, as plotted in Fig. 4, we identify two magnetic phases- the previously reported phase below $T_{\mathrm{N}}=104 \mathrm{~K}[18,25]$ and a new phase which persists up to $T_{\mathrm{N}}^{*} \sim 200 \mathrm{~K}$. We were unable to establish the exact transition temperature from ZF NMR alone, because the NMR signal-to-noise ratio deteriorates significantly at low resonance frequencies, close to the transition.

Measurements performed in $B_{0}=3.5 \mathrm{~T}$ did not reveal any static magnetic order above $T_{\mathrm{N}}$. However, as shown in Fig. 5, the dephasing rate of the nuclear spins $T_{2}^{-1}$ shows a significant hysteresis between $\sim 125$ and $200 \mathrm{~K}$. This almost coincides 


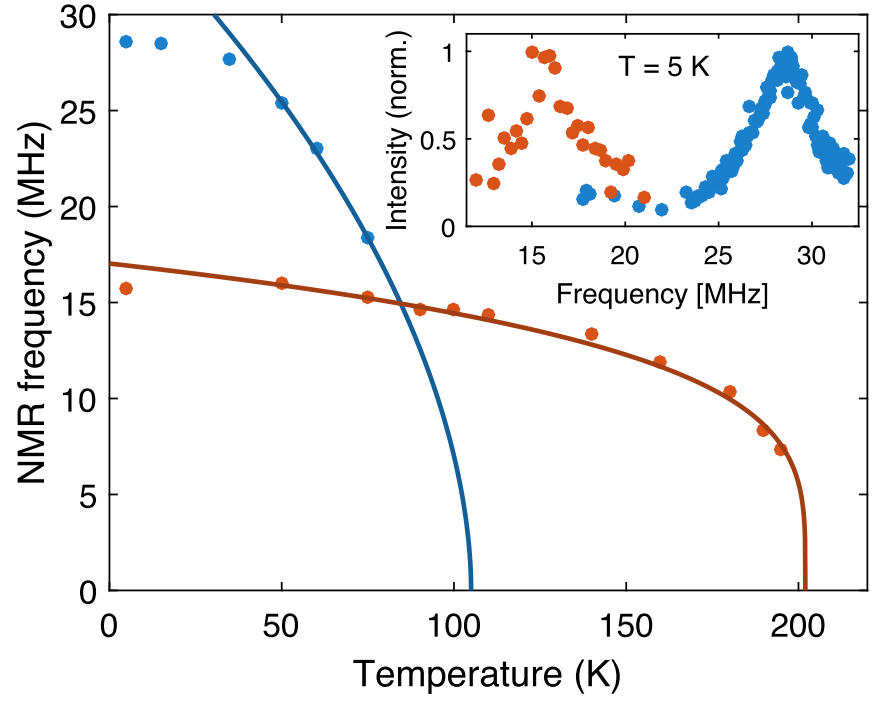

FIG. 4. Zero-field Tl NMR frequencies plotted against temperature, demonstrating the presence of static internal magnetic fields up to $\sim 200 \mathrm{~K}$. The solid lines are guides to the eye. Inset: Zero-field NMR spectra acquired at $5 \mathrm{~K}$. Two different sets of probe-head configurations and NMR pulse-sequence parameter sets were used to acquire the two parts of the spectrum, shown in blue and red. The intensities measured in two different setups are not directly comparable; hence signals are normalized to their maximal amplitude.

with the temperature range where we find the new magnetic phase in zero field, between $T_{\mathrm{N}}$ and $T_{\mathrm{N}}^{*}$. Below $100 \mathrm{~K}$, both the dephasing rate $T_{2}^{-1}$ and the relaxation rate $T_{1}^{-1}$ (not shown) follow an Arrhenius law $T_{i}^{-1}=\tau_{i} \exp \left(-\Delta / k_{\mathrm{B}} T\right)+C_{i}$ with an energy gap $\Delta / k_{\mathrm{B}}=82 \pm 5 \mathrm{~K}$ for the magnetic excitations.

Muon-spin rotation. Muon-spin rotation ( $\mu \mathrm{SR})$ experiments were performed in $\mathrm{TlNiO}_{3}$ powder in zero applied

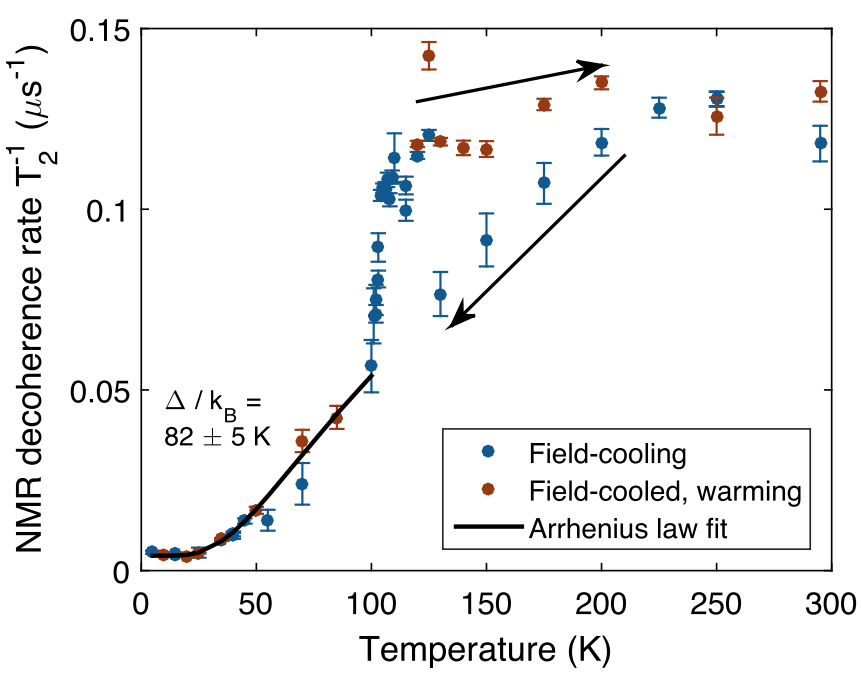

FIG. 5. Transverse NMR relaxation rate $T_{2}^{-1}$ in an applied magnetic field of several teslas, plotted against temperature. Note the large hysteresis between 125 and $200 \mathrm{~K}$. Below $100 \mathrm{~K}$, the relaxation rate follows an Arrhenius law with an activation energy $\Delta / k_{\mathrm{B}}=82 \pm 5 \mathrm{~K}$.

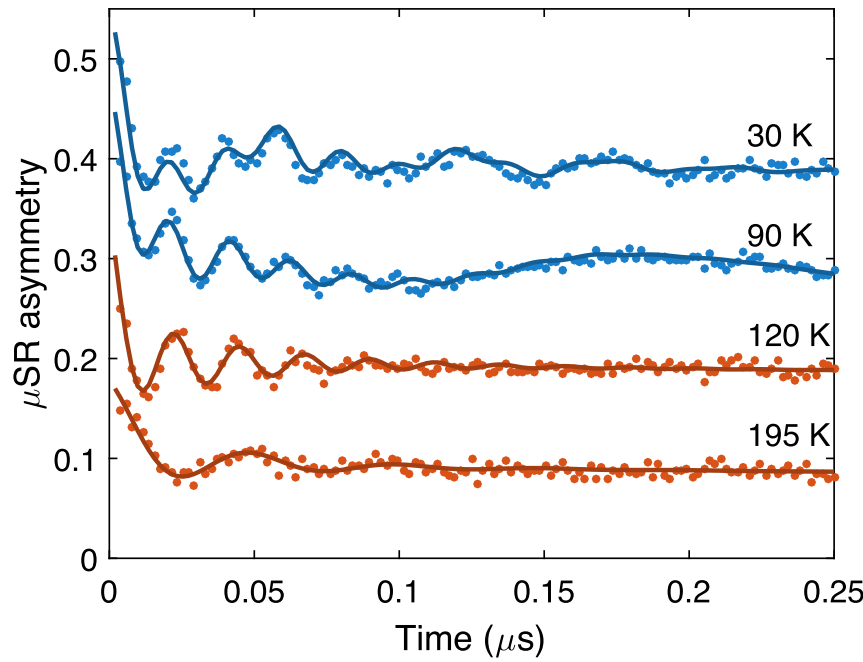

FIG. 6. Comparison of experimental zero-field $\mu$ SR asymmetries to the fits (solid line) described in the text body. Note that the oscillations mostly dephase within $0.2 \mu$ s, corresponding to a strong broadening of the frequency spectrum. Data acquired at different temperatures are offset vertically for clarity.

field between $5 \mathrm{~K}$ and ambient temperature using the General Purpose Spectrometer (GPS) at the Swiss Muon Source $(\mathrm{S} \mu \mathrm{S})$ at the Paul Scherrer Institut. In analogy to ZF NMR, the zero-field $\mu \mathrm{SR}$ frequencies reflect the local internal magnetic field (at the muon stopping site), hence providing a measure of the order parameter in the magnetically ordered phase. Unlike NMR, $\mu$ SR can detect low-frequency signals without loss of amplitude, as can be seen in Fig. 6. This allows for the determination of the new phase transition temperature $T_{\mathrm{N}}^{*}=202 \mathrm{~K}$ in zero field.

The time-domain histogram of detector $i, S_{i}(t)$, is given by

$$
S_{i}(t)=N_{0 i}\left[1+\operatorname{Re} A(t) e^{i \phi_{i}}\right] e^{-\frac{t}{\tau_{\mu}}}+B_{i},
$$

where $A(t)$ is the asymmetry, $N_{0 i}$ are normalized signal count rates, $\phi_{i}$ are phase offsets, $B_{i}$ are background count rates, and $\tau_{\mu}=2.2 \mu \mathrm{s}$ is the muon lifetime [27].

First, the $\phi_{i}$ are obtained from a calibration measurement in the PM phase in a transversely applied field of $3 \mathrm{mT}$. Second, the $N_{0 i}$ and $B_{i}$ are fitted for each dataset using Eq. (2) and assuming $A(t)$ to be constant, which is a good approximation at long times $t \gtrsim 0.3 \mu \mathrm{s}$. Then, the parameters of $A(t)$ are estimated by means of a global maximum-likelihood fit of the detector histograms. The Python [28] package iminuit [29] is used to perform the fits and determine the standard errors using the Minos algorithm [30].

Above $T_{\mathrm{N}}$, the expression

$$
A(t)=A\left[\frac{2}{3} \cos \left(2 \pi v^{(1)} t\right) e^{-\lambda_{\mathrm{x}}^{(1)} t}+\frac{1}{3} e^{-\lambda_{\mathrm{z}} t}\right]+B e^{-\lambda_{\mathrm{DC}} t}+C
$$

is used to fit the $\mu \mathrm{SR}$ asymmetry [27,31]. This expression accounts for two muon stopping sites: one where the mean local magnetic field is nonzero and one where it is zero. At both sites exponential relaxation occurs by fluctuations of the magnetic field. Exponential relaxation was also found in previous $\mu \mathrm{SR}$ experiments on other $\mathrm{RNiO}_{3}$ perovskites $[32,33]$ 


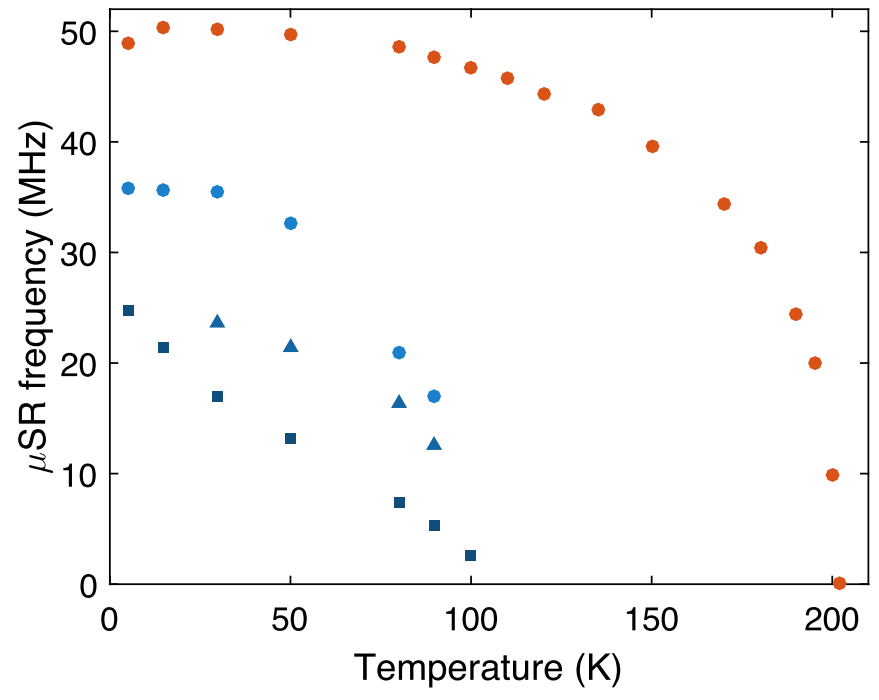

FIG. 7. Zero-field $\mu \mathrm{SR}$ frequencies $v^{(i)}$ in $\mathrm{TINiO}_{3}$ powder, as obtained from Eqs. (3) and (4). Parameters were fitted with a maximum-likelihood method using Minuit [30]. Different markers are assigned to different components of the $\mu \mathrm{SR}$ asymmetry. Error bars are omitted because they are smaller than the symbol size.

Below $T_{\mathrm{N}}$, the asymmetry is modeled using

$$
A(t)=a^{(0)} e^{-\lambda_{\mathrm{z}} t}+\sum_{i=1}^{4} a^{(i)} \cos \left(2 \pi v^{(i)} t\right) e^{-\lambda_{\mathrm{x}}^{(i)} t}+C,
$$

which describes the $\mu$ SR signal from four magnetically inequivalent muon sites experiencing nonzero local fields and exponential relaxation [27,31]. A comparison between the experimental asymmetry and our fits is shown in Fig. 6. Since the $\mu \mathrm{SR}$ signal dephases within the first $0.2 \mu \mathrm{s}$, the Fourier-transformed spectrum is strongly broadened. Thus it is impossible to resolve oscillations whose frequencies differ by less than a few MHz. This is the reason why we could establish only two frequencies at $100 \mathrm{~K}$ and only three frequencies at 5 and $15 \mathrm{~K}$. In the corresponding fits, a rapidly relaxing DC component was added phenomenologically to improve the fit convergence.

The temperature dependence of the frequencies $v^{(i)}$ plotted in Fig. 7 clearly shows the two phase transitions at $T_{\mathrm{N}}=104$ and $T_{\mathrm{N}}^{*}=202 \mathrm{~K}$. Additionally, one can see that there are four magnetically inequivalent muon sites below $T_{\mathrm{N}}$, giving rise to four different oscillation frequencies. The physical implication of these four muon sites is an open question. Since there are three inequivalent $\mathrm{O}^{2-}$ sites in $\mathrm{TlNiO}_{3}$ [18], a tentative explanation of the three frequencies appearing below $T_{\mathrm{N}}$ is that they originate from muons bound to the three different oxide ions. We have not been able to explain the approximately linear temperature-dependence of the lowest $\mu \mathrm{SR}$ frequency. However, this may be related to the occurrence of a magnetically ordered phase embedded within another ordered phase.

Conclusion. Our NMR and $\mu \mathrm{SR}$ data reveal the presence of a not-yet-reported magnetically ordered phase in $\mathrm{TlNiO}_{3}$ between $T_{\mathrm{N}}=104$ and $T_{\mathrm{N}}^{*}=202 \mathrm{~K}$ in low magnetic fields and confirm the previously known AFM phase below $T_{\mathrm{N}}$. Both phases clearly show static magnetic order on the time scales of NMR and $\mu \mathrm{SR}$. Due to the strong broadening of both the NMR and $\mu$ SR spectra, a distinction between short-range and long-range order is not possible. Future $\mu \mathrm{SR}$ experiments on $\mathrm{TINiO}_{3}$ are intended to map the phase boundary of the newly reported magnetic phase as a function of applied magnetic field and temperature and to explore the significance of the four $\mu \mathrm{SR}$ frequencies identified below $T_{\mathrm{N}}$. Further measurements on other compounds will show whether this new phase is unique to $\mathrm{TlNiO}_{3}$ or a universal feature of the $\mathrm{RNiO}_{3}$ family.

Acknowledgments. This work was financially supported in part by the Schweizerische Nationalfonds zur Förderung der Wissenschaftlichen Forschung (SNF). J.A.A. acknowledges the Spanish MINECO for funding the project MAT201341099-R.
[1] G. Demazeau, A. Marbeuf, M. Pouchard, and P. Hagenmuller, Sur une série de composés oxygènes du nickel trivalent derivés de la perovskite, J. Solid State Chem. 3, 582 (1971).

[2] M. L. Medarde, Structural, magnetic and electronic properties of $\mathrm{RNiO}_{3}$ perovskites $(\mathrm{R}=$ rare earth), J. Phys.: Condens. Matter 9, 1679 (1997).

[3] G. Catalan, Progress in perovskite nickelate research, Phase Trans. 81, 729 (2008).

[4] I. I. Mazin, D. I. Khomskii, R. Lengsdorf, J. A. Alonso, W. G. Marshall, R. M. Ibberson, A. Podlesnyak, M. J. Martínez-Lope, and M. M. Abd-Elmeguid, Charge Ordering as Alternative to Jahn-Teller Distortion, Phys. Rev. Lett. 98, 176406 (2007).

[5] H. Park, A. J. Millis, and C. A. Marianetti, Site-Selective Mott Transition in Rare-Earth-Element Nickelates, Phys. Rev. Lett. 109, 156402 (2012)

[6] A. Subedi, O. E. Peil, and A. Georges, Low-energy description of the metal-insulator transition in the rare-earth nickelates, Phys. Rev. B 91, 075128 (2015).

[7] J. Ruppen, J. Teyssier, O. E. Peil, S. Catalano, M. Gibert, J. Mravlje, J.-M. Triscone, A. Georges, and D. van der Marel,
Optical spectroscopy and the nature of the insulating state of rare-earth nickelates, Phys. Rev. B 92, 155145 (2015).

[8] J. L. García-Muñoz, J. Rodríguez-Carvajal, and P. Lacorre, Sudden appearance of an unusual spin density wave at the metal-insulator transition in the perovskites $\mathrm{RNiO}_{3}(\mathrm{R}=\mathrm{Pr}$, Nd), Europhys. Lett. 20, 241 (1992).

[9] J. L. García-Muñoz, J. Rodríguez-Carvajal, and P. Lacorre, Neutron-diffraction study of the magnetic ordering in the insulating regime of the perovskites $\mathrm{RNiO}_{3}(\mathrm{R}=\mathrm{Pr}$ and $\mathrm{Nd})$, Phys. Rev. B 50, 978 (1994).

[10] J. A. Alonso, J. L. García-Muñoz, M. T. Fernández-Díaz, M. A. G. Aranda, M. J. Martínez-Lope, and M. T. Casais, Charge Disproportionation in $\mathrm{RNiO}_{3}$ Perovskites: Simultaneous Metal-Insulator and Structural Transition in $\mathrm{YNiO}_{3}$, Phys. Rev. Lett. 82, 3871 (1999).

[11] M. T. Fernández-Díaz, J. A. Alonso, M. J. Martínez-Lope, M. T. Casais, and J. L. García-Muñoz, Magnetic structure of the $\mathrm{HoNiO}_{3}$ perovskite, Phys. Rev. B 64, 144417 (2001).

[12] V. Scagnoli, U. Staub, A. M. Mulders, M. Janousch, G. I. Meijer, G. Hammerl, J. M. Tonnerre, and N. Stojic, Role of magnetic 
and orbital ordering at the metal-insulator transition in $\mathrm{NdNiO}_{3}$, Phys. Rev. B 73, 100409(R) (2006).

[13] V. Scagnoli, U. Staub, Y. Bodenthin, M. García-Fernández, A. M. Mulders, G. I. Meijer, and G. Hammerl, Induced noncollinear magnetic order of $\mathrm{Nd}^{3+}$ in $\mathrm{NdNiO}_{3}$ observed by resonant soft x-ray diffraction, Phys. Rev. B 77, 115138 (2008).

[14] Y. Bodenthin, U. Staub, C. Piamonteze, M. García-Fernández, M. J. Martínez-Lope, and J. A. Alonso, Magnetic and electronic properties of $\mathrm{RNiO}_{3}(\mathrm{R}=\mathrm{Pr}, \mathrm{Nd}, \mathrm{Eu}, \mathrm{Ho}$ and $\mathrm{Y})$ perovskites studied by resonant soft $\mathrm{x}$-ray magnetic powder diffraction, J. Phys.: Condens. Matter 23, 036002 (2011).

[15] S.-W. Cheong and M. Mostovoy, Multiferroics: A magnetic twist for ferroelectricity, Nat. Mater. 6, 13 (2007).

[16] G. Giovannetti, S. Kumar, D. Khomskii, S. Picozzi, and J. van den Brink, Multiferroicity in rare-earth nickelates $\mathrm{RNiO}_{3}$, Phys. Rev. Lett. 103, 156401 (2009).

[17] A. Sakai, G. Zheng, and Y. Kitaoka, Strongly correlated metallic state of $\mathrm{LaNiO}_{3}:{ }^{139} \mathrm{La} \mathrm{NMR} \mathrm{study,} \mathrm{J.} \mathrm{Phys.} \mathrm{Soc.} \mathrm{Jpn.} \mathrm{71,} 166$ (2002).

[18] S. J. Kim, M. J. Martínez-Lope, M. T. Fernández-Díaz, J. A. Alonso, I. Presniakov, and G. Demazeau, Evidence of Ni(III) disproportionation in the $\mathrm{TlNiO}_{3}$ perovskite lattice through neutron powder diffraction and Mössbauer spectroscopy, Chem. Mater. 14, 4926 (2002).

[19] J. A. Alonso, M. J. Martínez-Lope, and I. Rasines, Preparation, crystal structure, and metal-to-insulator transition of $\mathrm{EuNiO}_{3}$, J. Solid State Chem. 120, 170 (1995).

[20] J. A. Alonso, M. J. Martínez-Lope, M. T. Casais, M. A. G. Aranda, and M. T. Fernández-Díaz, Metal-insulator transitions, structural, and microstructural evolution of $\mathrm{RNiO}_{3}(\mathrm{R}=\mathrm{Sm}, \mathrm{Eu}$, Gd, Dy, Ho, Y) perovskites: Evidence for room-temperature charge disproportionation in monoclinic $\mathrm{HoNiO}_{3}$ and $\mathrm{YNiO}_{3}$, J. Am. Chem. Soc. 121, 4754 (1999).

[21] J. A. Alonso, M. J. Martínez-Lope, M. T. Casais, J. L. García-Muñoz, M. T. Fernández-Díaz, and M. A. G. Aranda, High-temperature structural evolution of $\mathrm{RNiO}_{3}(\mathrm{R}=\mathrm{Ho}, \mathrm{Y}$, Er, Lu) perovskites: Charge disproportionation and electronic localization, Phys. Rev. B 64, 094102 (2001).

[22] J. A. Alonso, M. J. Martínez-Lope, I. A. Presniakov, A. V. Sobolev, V. S. Rusakov, A. M. Gapochka, G. Demazeau, and
M. T. Fernández-Díaz, Charge disproportionation in $\mathrm{RNiO}_{3}$ ( $\mathrm{R}=\mathrm{Tm}, \mathrm{Yb}$ ) perovskites observed in situ by neutron diffraction and ${ }^{57} \mathrm{Fe}$ probe Mössbauer spectroscopy, Phys. Rev. B 87, 184111 (2013).

[23] J. A. Alonso, M. J. Martínez-Lope, M. T. Casais, J. L. Martínez, G. Demazeau, A. Largeteau, J. L. García-Muñoz, A. Muñoz, and M. T. Fernández-Díaz, High-pressure preparation, crystal structure, magnetic properties, and phase transitions in $\mathrm{GdNiO}_{3}$ and $\mathrm{DyNiO}_{3}$ perovskites, Chem. Mater. 11, 2463 (1999).

[24] A. Muñoz, J. A. Alonso, M. J. Martínez-Lope, and M. T. Fernández-Díaz, On the magnetic structure of $\mathrm{DyNiO}_{3}$, J. Solid State Chem. 182, 1982 (2009).

[25] S.-J. Kim, G. Demazeau, J. A. Alonso, and J.-H. Choy, High pressure synthesis and crystal structure of a new Ni(III) perovskite: $\mathrm{TlNiO}_{3}$, J. Mater. Chem. 11, 487 (2001).

[26] Y. Yamada and A. Sakata, An analysis method of antiferromagnetic powder patterns in spin-echo NMR under external fields, J. Phys. Soc. Jpn. 55, 1751 (1986).

[27] A. Yaouanc and P. Dalmas de Réotier, Muon Spin Rotation, Relaxation, and Resonance (Oxford University Press, New York, 2011).

[28] Python Software Foundation, Python (version 2.7.11) [http://www.python.org].

[29] iminuit - MINUIT from Python (version 1.2), [http://github.com/iminuit/iminuit].

[30] F. James and M. Roos, Minuit—a system for function minimization and analysis of the parameter errors and correlations, Comput. Phys. Commun. 10, 343 (1975).

[31] P. Dalmas de Réotier and A. Yaouanc, Muon spin rotation and relaxation in magnetic materials, J. Phys.: Condens. Matter 9, 9113 (1997).

[32] J. L. García-Muñoz, P. Lacorre, and R. Cywinski, Muon-spinrelaxation study of magnetic order in $\mathrm{RNiO}_{3}(\mathrm{R}=$ rare earth) below the metal-insulator transition, Phys. Rev. B 51, 15197 (1995).

[33] J. L. García-Muñoz, R. Mortimer, A. Llobet, J. A. Alonso, M. J. Martínez-Lope, and S. P. Cottrell, $\mu$ SR study of short-range charge order in $\mathrm{YNiO}_{3}$ above the monoclinic-orthorhombic transition, Physica B (Amsterdam, Neth.) 374-375, 87 (2006). 